\title{
Abstracts of Award-Winning Posters, 18th Annual Health Sciences Poster Conference, Faculty of Medicine, Health Sciences Centre, Kuwait University, Kuwait, May 7-9, 2013
}

\section{Dr. Nael Al-Naqeeb Undergraduate Research Award}

\author{
1 \\ Neuron and Astrocyte Protection against Lead \\ Toxicity by (-)-Epigallocatechin-3-Gallate in \\ Hippocampal Primary Cell Culture \\ H.M. Al Shimali ${ }^{b}$, M.S. Rao ${ }^{a}$, W.M. Renno ${ }^{a}$, S. Smitha ${ }^{a}$ \\ ${ }^{a}$ Department of Anatomy, ${ }^{b}$ Faculty of Medicine, \\ Kuwait University, Kuwait
}

Introduction: Epigallocatechin-3-gallate (EGCG), the most active component of Green tea, has been shown to have neuroprotective and antioxidant properties. The objective of the present study was to investigate the role of EGCG in protection of newly generated neurons and astrocytes against lead-induced toxicity in hippocampal cell culture. Methods: A primary culture of fetal hippocampal tissue (E19) was done in DMEM medium. On day 3, cultures were divided into four groups and treated as follows for 4 days: (1) normal control (NC, $\mathrm{n}=6$ ) - no treatment; (2) lead only $(\mathrm{LO}, \mathrm{n}=6)$ - medium was replaced with medium containing 20 $\mu \mathrm{M}$ lead nitrate; (3) EGCG $(\mathrm{n}=6)$ - medium was replaced with medium containing $50 \mu \mathrm{M}$ EGCG, and (4) lead + EGCG, (L + EGCG, $\mathrm{n}=6$ ) - medium was replaced with medium containing 20 $\mu \mathrm{M}$ lead nitrate and $50 \mu \mathrm{M}$ EGCG. Cell survival was analyzed by counting number of cells in randomly selected fields. Culture dishes were stained for doublecortin and glial fibrillary acidic protein (GFAP) immunostaining, and the number of stained neurons and astrocytes in the culture were quantified. Data were analyzed with one-way ANOVA and Bonferroni test. Results: The LO cultures showed a significantly decreased number of surviving cells compared to the NC, EGCG, and L + EGCG groups. Cells in the LO group had fewer and shorter processes. The cell death count was significantly decreased $(40 \%, \mathrm{p}<0.001)$ in cultures treated with $\mathrm{L}$ + EGCG compared to the LO group. The cultures treated with L + EGCG showed a significantly increased number of doublecortin positive neurons $(40 \%, \mathrm{p}<0.001)$ and GFAP immunopositive astrocytes $(50 \%, \mathrm{p}<0.001)$ compared to the LO group. Conclusions: These data suggest that EGCG can counteract lead toxicity and enhance cell survival and differentiation into neurons and astrocytes in culture.

Funding agency: Kuwait University Grant No. GM01/01\&GM 01/05. We would like to thank Ms. Sunitha Pramod in the Research Core Facility for her technical assistance.

\section{2}

Nigella sativa Seeds Extract Protects Hippocampal Neurons, Enhances Neurogenesis and Increases Astrocytes in a Kainic Acid Model of Temporal Lobe Epilepsy

O.M. Hedaya ${ }^{a}$, M.S. Rao ${ }^{b}$, W.M. Renno ${ }^{b}$, S. Smitha ${ }^{b}$

${ }^{a}$ Faculty of Pharmacy and bepartment of Anatomy, Faculty of Medicine, Kuwait University, Kuwait

Introduction: Recent studies have shown that the extracts of Nigella sativa seeds have many therapeutic effects. The present study objective was to investigate the effect of an alcoholic extract of $N$. sativa (NSE) on hippocampal neurons, astrocytes and neurogenesis in a kainic acid model of temporal lobe epilepsy (TLE). Methods: TLE was developed by injecting kainic acid $(0.25 \mu \mathrm{g})$ into lateral ventricles in adult Wistar rats. Rats were then divided into the following groups: (1) lesion only ( $\mathrm{LO}, \mathrm{n}=12$, received daily $1 \mathrm{ml}$ of saline orally for $48 \mathrm{~h}$ or 1 week) and (2) lesion + NSE $[\mathrm{L}+\mathrm{NSE}, \mathrm{n}=12$, received daily NSE $(400 \mathrm{mg} / \mathrm{kg})$ in $1 \mathrm{ml}$ of saline orally for $48 \mathrm{~h}$ or 1 week]. Cryostat brain sections were processed for cresyl violet, Flurojade-B, doublecortin (DCX) and glial fibrillary acidic protein (GFAP) immunostaining. The number of sur-

\begin{tabular}{ll}
\hline KARGER & $\begin{array}{l}\text { @ } 2013 \mathrm{~S} . \text { Karger AG, Basel } \\
\text { 1011-7571/13/0226-0606 } \$ 38.00 / 0 \quad \text { Karger } \\
\text { Open cesess }\end{array}$ \\
$\begin{array}{l}\text { E-Mail karger@karger.com } \\
\text { www.karger.com/mpp }\end{array}$ & $\begin{array}{l}\text { This is an Open Access article licensed under the terms of the } \\
\text { Creative Commons Attribution-NonCommercial 3.0 Un- } \\
\text { ported license (CC BY-NC) (www.karger.com/OA-license), } \\
\text { applicable to the online version of the article only. Distribu- } \\
\text { tion permitted for non-commercial purposes only. }\end{array}$
\end{tabular}


viving, degenerating (Flurojade), newly generated neurons (DCX) and astrocytes (GFAP) was quantified in the dentate hilus (DH) and CA3 regions of the hippocampus. Data were analyzed with one-way ANOVA followed by Bonferroni test. Results: At $48 \mathrm{~h}$, Flurojade-B staining revealed a significantly lower number ( 20\%) of degenerating neurons in $\mathrm{DH}$ and CA3 regions in the $\mathrm{L}+\mathrm{NSE}$ group compared to the LO group ( $\mathrm{p}<0.05)$. At 1 week, a significantly lower $(\sim 60 \%)$ number of degenerating neurons was found in the L + NSE compared to the LO group ( $\mathrm{p}<0.0001)$. DCX staining showed significantly increased neurogenesis $(\sim 30 \%)$ in the dentate gyrus after 1 week of treatment in the L + NSE group $(\mathrm{p}<$ $0.001)$. GFAP staining revealed significantly increased astrocytes in the $\mathrm{DH}$ and $\mathrm{CA} 3$ region after 1 week of treatment. Conclusions: These data suggest that NSE can prevent the kainic acid-induced neurodegeneration and enhances neurogenesis in TLE.

Funding agency: Kuwait University Grant No. GM01/01\&GM $01 / 05$. We would like to thank Ms. Sunitha Pramod in the Research Core Facility for her technical assistance.

\section{Best Postgraduate Awards}

\section{Graduate MSc}

\section{1}

\section{Lead Imparts Cytotoxic and Mitogenic Effects on Hepatocytes in a Dose- and Time-Dependent Manner in the Rat Liver}

\section{L.M. Mujaibel ${ }^{b}$, K. Narayana $^{a}$}

aDepartment of Anatomy, Faculty of Medicine, and

${ }^{b}$ Environmental Science, College of Graduate Studies,

Kuwait University, Kuwait

Introduction: Lead exposure is increasingly becoming a serious public health concern. Recent reports indicate that lead may act as a weak mutagen or carcinogen. The carcinogenic potential of any carcinogen depends on its ability to induce irreparable DNA damage and uncontrolled cell proliferation. However, mitogenic effects of lead on hepatocytes are not well studied. With this in view, the objective of the present study was to investigate a putative hepatocyte proliferation in lead-exposed liver. Methods: Adult Wistar rats (13-15 weeks old; $\mathrm{n}=8$ ) were exposed via drinking water to $0,0.5$, and $1 \%$ lead acetate for 3,14 , and 35 days, and the livers were sampled the next day. Histopathological changes in the liver were evaluated in toluidine blue-stained thick sections. The expression of PCNA and Ki-67 was evaluated by Western blotting and confocal microscopy. Data were analyzed by one-way ANOVA followed by least significant difference and $\mathrm{p}<0.05$ was considered significant. Results: Lead exposure resulted in structural changes in livers such as margination of mitochondria, dissolution of glycogen, vacuolation, sinusoidal swelling, and degeneration of hepatocytes. On all sampling days, PCNA activities increased, but in a dose-dependent manner on day 15 and 36, compared to that in control livers $(\mathrm{p}<0.05)$. In $0.5 \%$ dose groups, a time-dependent decrease and in $1 \%$ dose groups, a time-dependent increase in the protein level was observed ( $\mathrm{p}<0.05)$. The Ki-67 expression also increased on all days. A time-dependent increase in the expression was observed on day $36(\mathrm{p}<0.05)$. Conclusions: Lead exposure induces concomitant hepatocyte degeneration and proliferation in the liver. Thus, lead simultaneously acts as a cytotoxic agent and a mitogen for hepatocytes. Long-term lead exposure may stimulate hepatic carcinogenesis. On the other hand, upregulated PCNA expression may also be involved in DNA damage repair pathways as lead is known to induce DNA damage.

Funding agency: Kuwait University Grant No. YS05/09, GM01/01, and GM01/05.

\section{2}

\section{Profiling the Expression of 84 Genes Related to the Th17 Regulatory Network in Systemic Lupus Erythematosus Patients}

\section{A.T. Al-Failakawi' , S.M. Al-Fadhlib , A.G. Al-Muhanac}

${ }^{a}$ Department of Biochemistry, Kuwait University Faculty of Sciences, bepartment of Medical Laboratory Sciences, Kuwait University Faculty of Allied Health Sciences, and 'Department of Rheumatology, Ministry of Health Mubarak Al-Kabeer Hospital, Kuwait

Introduction: Systemic lupus erythematosus (SLE) is a connective-tissue autoimmune-mediated disorder, marked by a wide array of organ system dysfunctions and with various clinical presentations. The mechanism of its pathogenesis is unclear. T helper (Th) cells have a big role in the pathogenesis of autoimmune diseases. Recently, many pieces of evidence have emerged showing that inappropriate regulation of type $17 \mathrm{Th}$ cells (Th17) plays a fundamental role in the development of many autoimmune diseases including SLE. Methods: Ninety-six study subjects, 66 SLE patients and 30 age- and sex-matched healthy controls, were recruited. Th17 autoimmunity and inflammation RT-PCR array was used for analyses of Th17-related gene expression in peripheral white blood cells from collected samples. IL-17A and IL-17F concentrations were measured in sample serum using ELISA technique. Web-based PCR array data analysis software (SABiosciences) and GraphPad Prism V.6 software were used for data analysis. Results: Out of the 84 targets, 14 genes (IL-17A, IL-17C, IL-17D, IL-17F, IL-18, IL12RB2, IL12R, CCL2, CCL20, CXCL5, MMP3, RORC, STAT4, and TRAF6) were found to be differently expressed in SLE patients and healthy controls. These genes showed statistically significant $(\mathrm{p}<0.0006)$ changes and were downregulated $(\mathrm{FC}<1)$. No significant difference was observed between SLE patients with and without nephritis. Serum concentrations of IL17A showed a difference in SLE compared to controls $(\mathrm{p}=0.032)$, and IL-17F showed a difference in SLE compared to healthy controls $(\mathrm{p}<0.0001)$. Conclusions: To our knowledge, our study is the first to confirm the association of Th17 regulatory network genes with SLE in patients of Arab ethnicity. The difference in Th17 signaling pathway genes indicates that this pathway may be involved in the pathogenesis of SLE.

Funding agency: College of Graduate Studies. 


\section{Graduate PhD}

1

\section{Rapid and Accurate Identification of Candida dubliniensis and Candida albicans by Real-Time PCR and Melting Curve Analysis}

\author{
M. Asadzadeh, S. Ahmad, A. Theyyathel, Z.U. Khan \\ Department of Microbiology, Faculty of Medicine, \\ Kuwait University, Kuwait
}

Introduction: Candida dubliniensis shares many phenotypic features with Candida albicans and may be misidentified in clinical mycology laboratories. Molecular methods are preferred over phenotypic tests for unambiguous differentiation of $C$. dubliniensis from C. albicans. The aim of this study was to develop a duplex real-time PCR (RT-PCR) that could differentiate these two species via a melting curve analysis. Methods: Two species-specific primer pairs targeting rDNA sequences were designed. The LightCycler Fast Start DNA Master SYBR Green I kit and instrument were used for amplification and melting curve analysis as directed by the manufacturer. Amplification products were also analyzed by agarose gel electrophoresis (AGE) to confirm RT-PCR results. The assay was evaluated on 226 clinical isolates including $C$. albicans $(\mathrm{n}=113)$, C. dubliniensis $(\mathrm{n}=98)$, and 9 other Candida spp. previously identified by Vitek 2 yeast identification system and/or direct DNA sequencing of rDNA. Results: The melting points (Tm) for reference C. albicans and C. dubliniensis strains were 86.55 and $82.75^{\circ} \mathrm{C}$, respectively. Analysis of RT-PCR products by AGE confirmed amplification of expected DNA fragments for both C. albicans and C. dubliniensis, and no primer dimer artifacts were visible. When RT-PCR was applied on 226 clinical isolates, the measured Tm values for C. albicans $(\mathrm{n}=113)$ and C. dubliniensis $(\mathrm{n}=$ 98) were $86.68+0.529$ and $82.616+0.535^{\circ} \mathrm{C}$, respectively, while no amplification was detected from other Candida species. Conclusions: The RT-PCR assay using SYBR Green dye for melting point analysis is a rapid and reliable test for detection and differentiation of C. dubliniensis or C. albicans strains. The assay does not require prior identification of clinical isolates as $C$. dubliniensis/C. albicans strains by germ tube formation, and the whole procedure could be completed within $2 \mathrm{~h}$.

Funding agency: Kuwait University Research Administration Grant YM 10/11.

\section{Case Report Award}

\section{Vernix Caseosa Peritonitis after Vaginal Delivery - A Case Report}

\author{
S.A.Sadath ${ }^{b}$, F.I. Abo Diba ${ }^{b}$, S. Nayak, I. Al Shamalib, \\ M.F.E. Diejomaoh ${ }^{a, b}$
}

aDepartment of Obstetrics and Gynecology, Faculty of Medicine, Kuwait University, and Departments of ${ }^{\mathrm{b}}$ Obstetrics and

Gynecology, and 'Pathology, Maternity Hospital, Kuwait

Background: Vernix caseosa peritonitis (VCP) is a very unusual complication caused by inflammatory response to amniotic fluid spilled into the maternal peritoneal cavity. Only 23 cases of this entity have been reported and all occurred after caesarean section. Case Summary: This is the first case of VCP reported following vaginal delivery. Mrs. A., 28 years old, $\mathrm{P} 2+0+0+2$, with one previous caesarean section, was admitted at 41 weeks gestation in active labor. Antenatal care in the current pregnancy was uneventful. Labor progressed rapidly to the second stage, and because of repeated fetal decelerations, vacuum extraction was performed to deliver a healthy male baby, $4.410 \mathrm{~kg}$, Apgar scores 7, 8. The mother developed fever, acute abdominal pain, and distension about $3 \mathrm{~h}$ after delivery. Physical examination revealed a temperature of $37.9^{\circ} \mathrm{C}$, pulse was $118 / \mathrm{min}$, blood pressure $105 / 70 \mathrm{~mm} \mathrm{Hg}$, and the abdomen was slightly distended with generalized diffuse tenderness; pelvic examination was negative. A diagnosis of acute abdomen was made. Hemoglobin was $12.9 \mathrm{~g} / \mathrm{dl}$ and white blood cells 10.6 with polymorphonuclear leukocytosis; ultrasound demonstrated some free fluid in the pelvis and abdomen. Laparotomy was performed soon afterwards and it revealed neither uterine scar rupture nor other surgical emergencies, but $500 \mathrm{ml}$ of turbid fluid and some cheesy material on the serosal surface of all viscera; tissue biopsy was obtained from the cheesy material and adjacent glands. The patient had a course of antibiotics; her postoperative period was uneventful and her recovery complete. Histology of the peritoneal fluid and tissue biopsy resulted in a diagnosis of VCP. Conclusion: Clinical diagnosis of peritonitis due to vernix caseosa should be considered in patients presenting postpartum with an acute abdomen. Characteristic intraoperative findings and the involvement of a pathologist can promote a more conservative approach and prevent unnecessary invasive procedures. 